\title{
Atomic displacement in solids: analysis of the primary event and the collision cascade. Part I: Neutron and positive ion irradiation
}

\author{
P de Almeida ${ }^{1}$ and J Räisänen \\ Accelerator Laboratory, PO Box 43, FIN-00014 University of Helsinki, Finland \\ E-mail: p.dealmeida@lr.tudelft.nl
}

Received 12 November 2004, in final form 18 January 2005

Published 17 March 2005

Online at stacks.iop.org/EJP/26/371

\begin{abstract}
A modern, mathematical-physics introduction to the analytical problem of atomic displacement in solids which is both technically adequate and relevant to an introductory graduate students' curriculum in radiation damage theory is reported. The problematic of atomic displacement in solids is introduced didactically, deriving first the primary event (that is, the formation of the primary knock-on atom) and then building on that specific set of results in order to extend their basics to secondary, tertiary and higher-order progenythe collision cascade-for both neutrons and positive ions, namely, their average damage functions, displacement cross-sections and energy spectra. A comparison of atomic displacement in solids under neutron and positive ion irradiation is discussed in terms of the physical concept of concentration of displaced atoms (or displacement dose).
\end{abstract}

\section{Introduction}

Atomic displacement in solids is a specific topic in the realm of radiation damage studies and has been covered in many different ways, both theoretically and experimentally, during the past 40-50 years within a relatively small international community, for instance, in the frame of mechanical properties degradation of structural materials used in the construction of a nuclear fission reactor. In a rather different context, though, ion-accelerators have been used during the same period of time and covered many of the more basic aspects related to atomic

1 Present address: Fundamentals of Advanced Materials, Faculty of Aerospace Engineering, Delft University of Technology, Kluyverweg 1, NL-2629 HS Delft, The Netherlands. 
displacement in solids, for instance, in the frame of irradiation induced phase transformations and surface property modification.

This paper relates closely to the topics on which we have spent a significant portion of our professional lives and provides a modern, mathematical-physics introduction to the analytical problem of atomic displacement in solids under neutron or positive ion irradiation that is technically adequate and relevant to an introductory graduate students' curriculum in radiation damage theory. Having this objective in mind, we will introduce the problem of atomic displacement in solids deriving first the primary event and then building on this specific set of results in order to extend their basics to secondary, tertiary and higher-order progenythe collision cascade - for both neutrons and positive ions. A rather pertinent phenomenon intimately related to atomic displacement production is defect recombination (or annealing) and it will only be mentioned here very briefly during the discussion, despite its importance (see, for instance, Anno (1985)).

When a high energy incident particle penetrates a solid, the kinetic energy of this particle is dissipated by a certain number of mechanisms. In the case of charged particles (i.e., electrons, protons, alpha particles, positive ions) the dominant energy loss mechanism involves energy transfers from the incident particle to the electrons of the solid target. The electrons may be excited to higher energy levels, with subsequent decay, or possibly ionization.

From the point of view of radiation damage in solids, ionization effects are important in organic materials, ionic crystals and semiconductors, but may be essentially ignored in metals. In this paper attention is initially focused mainly on the interaction of the incident particle with the nuclei of the solid target; later the fate of the atoms excited by the incident particle or by collision with other atoms in the solid is described.

The simplest disturbance of the crystalline lattice is that which involves the position of one atom only, generally referred to as a point defect, and despite the existence of three main types, the unoccupied lattice site or vacancy is the most common. In many aspects the interstitial atom or simply interstitial is the analogue of the vacancy. A stable interstitialvacancy pair is defined as a Frenkel pair (or defect) though it should be clear that no one interstitial is necessarily associated with a particular vacancy. In any given crystalline lattice, to a greater or lesser degree, foreign atoms or impurity atoms are present which could either occupy interstitial or substitutional lattice site positions. In the case of impurities produced by fission neutrons (transmutation) their number will be insignificant when compared with the number of Frenkel pairs which derive from atomic displacements in the solid.

For an introduction to point defect complexes (crowdion, split interstitial, colour centres, etc), defect clusters (of interstitials or vacancies) and extended defects (dislocations, stacking faults, etc) refer to, for instance, Chadderton (1965).

\section{Analysis of the primary event}

The formulation of the interaction of primary particles and atoms described here follows that of Thompson (1969) which is somewhat exotic but very consistent and the more conventional one appearing in, for instance, Dienes and Vineyard (1957) which is more self-contained and largely adopted in the literature. The interactions between incident particles and atoms of a solid, in the energy range of interest, occur essentially as isolated events. Thus, the history of an incident particle through a solid may generally be thought of as a succession of collisions with atoms in the solid. In each such collision a portion of the kinetic energy of the incident particle is transferred to the struck atom. This event can be treated as a two-body collision provided that the mean free path between collisions is much greater than the interatomic spacing of the solid. The probability of correlation effects owing to neighbouring atoms 


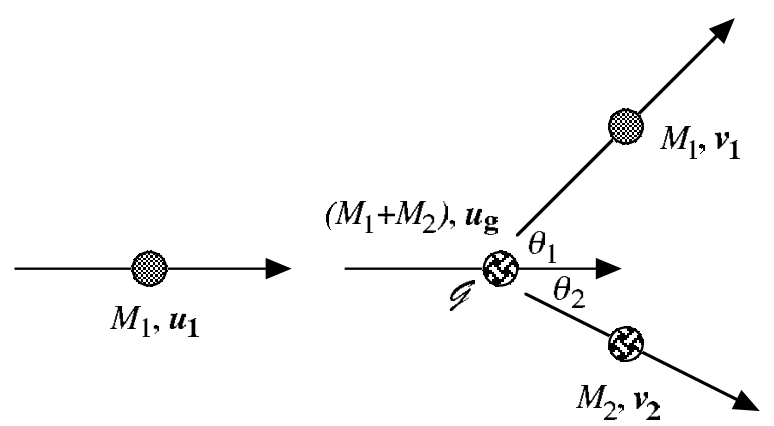

Figure 1. A two-body collision in laboratory $(\mathcal{L})$ coordinates.

recoiling almost simultaneously is then very small. The system treated here will satisfy this condition, but eventually multiple collisions will be considered in the next section.

The momentum of the recoiling atom is the parameter which determines the damage to the solid target and it will be our aim to calculate it. The interaction of radiation with matter is generally considered from the viewpoint of the scattered particle, here however we shall be most concerned with the energy of the recoil atom and the angle between its path and that of the incident particle. First let us derive some general physics governing the two-body collisions considering only the asymptotic values of momentum at great distances from the collision. Under this specific condition there is no violation of quantum-mechanical laws in assigning a precise momentum to a particle since we are not localizing its position along the path. The principles of conservation of momentum and energy are all that are necessary to evaluate the recoil energy as a function of the recoil angle. Initially we shall assume that all collisions are elastic and, furthermore, that velocities are small enough for non-relativistic mechanics to hold.

Let the mass, velocity and energy of the incident particle be $M_{1}, \boldsymbol{u}_{1}$ and $E_{1}$ before the collision; the angle through which it is scattered $\theta_{1}$ and its final velocity $\boldsymbol{v}_{1}$. Let $M_{2}$ be the mass of the struck particle, $\boldsymbol{v}_{2}$ its final velocity and $\theta_{2}$ the angle of recoil (figure 1 ).

Some simplification is brought about by treating the motion relative to the centre of mass $\mathcal{G}$ in what will be referred to as $\mathcal{G}$ coordinates. The coordinate system at rest in the laboratory will be called the $\mathcal{L}$ system. The velocity $\boldsymbol{u}_{g}$ of $\mathcal{G}$ in the $\mathcal{L}$ coordinates is found simply by assuming a particle of mass $\left(M_{1}+M_{2}\right)$ to be travelling with a velocity $\boldsymbol{u}_{g}$ and equating its momentum to that of the incident particle: $M_{1} \boldsymbol{u}_{1}$. Hence we have

$$
u_{g}=\frac{M_{1} u_{1}}{M_{1}+M_{2}} \text {. }
$$

By subtracting this vector from all velocities in the problem we transform from $\mathcal{L}$ to $\mathcal{G}$ coordinates, as shown in figure 2 where the meaning of $V_{1}, V_{2}, U_{1}$ and $\phi$ are defined.

Because $\mathcal{G}$ must remain at rest, $V_{1}$ and $V_{2}$ are in opposite directions. A further simplification arises from the conservation of energy and momentum which require:

$$
\begin{aligned}
& M_{1} U_{1}^{2}+M_{2} u_{g}^{2}=M_{1} V_{1}^{2}+M_{2} V_{2}^{2}, \\
& M_{1} U_{1}+M_{2} u_{g}=M_{1} V_{1}+M_{2} V_{2} .
\end{aligned}
$$

In $\mathcal{L}$ coordinates the equations would include trigonometric functions and would not possess the simple form of this pair which can be simultaneously satisfied if and only if each particle leaves $\mathcal{G}$ at the same speed with which it approached, namely:

$$
U_{1}=V_{1} \quad \text { and } \quad u_{g}=V_{2} .
$$



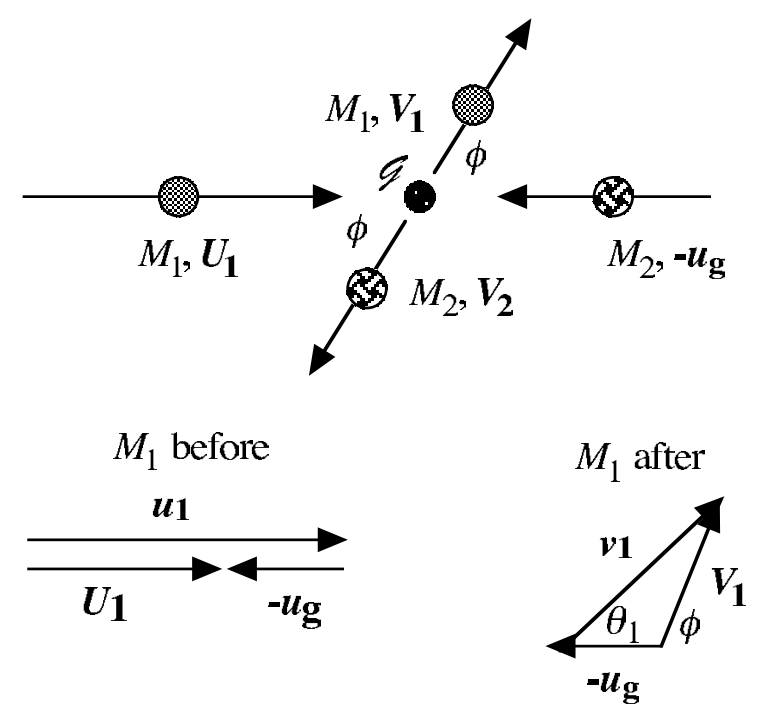

$M_{2}$ before

$M_{2}$ after
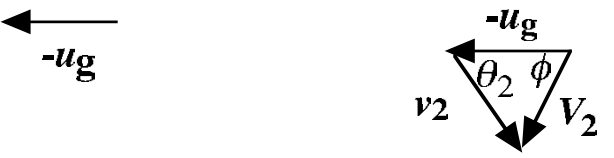

Figure 2. The transformation to centre of mass $(\mathcal{G})$ coordinates.

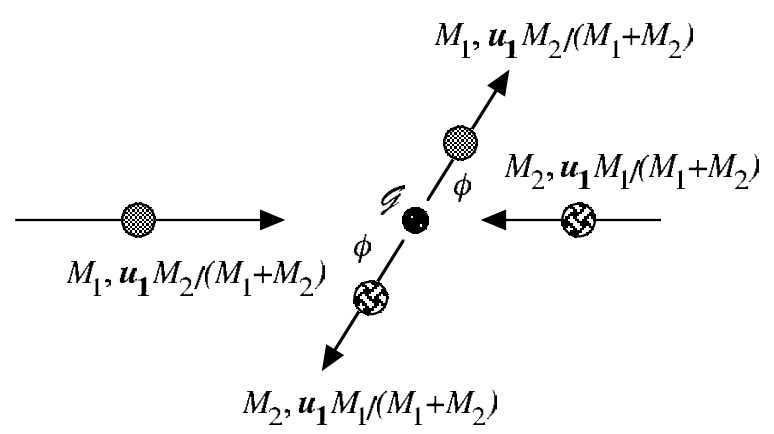

Figure 3. The two-body collision in centre of mass $(\mathcal{G})$ coordinates.

Then, calculating $V_{1}$ from figure 2 and equation (1):

$$
V_{1}=\frac{M_{2} u_{1}}{M_{1}+M_{2}}
$$

the simplified collision is represented in figure 3.

An important quantity is the total kinetic energy, either before or after collision, in the $\mathcal{G}$ system. This is easily shown to be equal to

$$
\frac{M_{2} E_{1}}{M_{1}+M_{2}} .
$$

It is easily verified, too, that this term, when added to the kinetic energy of the centre of mass $\frac{1}{2}\left(M_{1}+M_{2}\right) u_{g}^{2}$ is just $E_{1}$ confirming that the total energy of the two bodies remains unchanged 
by the new description in $\mathcal{G}$ coordinates. We require the recoil energy $E_{2}$ in $\mathcal{L}$ coordinates and must therefore calculate $v_{2}$ from the appropriate vector triangle in figure 2 using the cosine law to yield:

$$
v_{2}^{2}=\frac{2(1-\cos \phi) M_{1}^{2} u_{1}^{2}}{\left(M_{1}+M_{2}\right)^{2}}
$$

and hence

$$
E_{2}=\Lambda E_{1} \sin ^{2}(\phi / 2), \quad \text { with } \quad \Lambda=\frac{4 M_{1} M_{2}}{\left(M_{1}+M_{2}\right)^{2}}
$$

In this context $\Lambda$ has special meaning for when $\phi=\pi$ (head-on collision in classical terms) and particles approach and recede along one axis, we have the maximum possible energy transfer with

$$
\hat{E}_{2}=\Lambda E_{1} \text {. }
$$

Obviously, when $\phi=0$, no scattering occurs and $E_{2}=0$ as we would expect. In any radiation damage problem there will be a minimum recoil energy, $\check{E}_{2}$, which is just capable of producing displacement damage. There is also a minimum bombarding energy $\breve{E}_{1}$ which has $\breve{E}_{2}$ as its maximum possible recoil energy, namely:

$$
\check{E}_{2}=\Lambda \check{E}_{1} \text {. }
$$

The minimum energy necessary for the displacement of an atom depends, for a certain solid, on a number of factors discussed in detail later on. We merely note that the direction of the struck atom's initial momentum, with respect to the lattice, may be an important factor (see, for instance, Sosin and Bauer (1969), p 153). For the sake of simplicity, we assume here that directionality is of reduced importance and that we may refer to a threshold (minimum) displacement energy, $\check{E}_{2}$, independent of direction. Clearly, we are only concerned with particles for which $E_{1}>\check{E}_{1}$.

The relationship between $\theta_{2}$ and $\phi$ is obtained by considering the components of $M_{2}$ 's velocity parallel and perpendicular to the axis of incidence. The values in $\mathcal{L}$ coordinates must equal those in $\mathcal{G}$ coordinates when the appropriate components of $\boldsymbol{u}_{\boldsymbol{g}}$ are added, namely:

$$
V_{2} \cos \phi+v_{2} \cos \theta_{2}=u_{g}, \quad V_{2} \sin \phi=v_{2} \sin \theta_{2}
$$

Hence using equation (4) for $V_{2}$ we obtain

$$
\tan \theta_{2}=\frac{\sin \phi}{(1-\cos \phi)} .
$$

Before being able to calculate the number of recoils in the energy interval $\mathrm{d} E_{2}$ at $E_{2}$ we must know the angular distribution function which yields the probability of recoil into $\mathrm{d} \phi$ at $\phi$. This can only be obtained with a detailed knowledge of the interaction forces between particles $M_{1}$ and $M_{2}$. Also, before applying classical mechanics two criteria must be satisfied, namely, $\lambda \ll a$ which means that the particle trajectories must be well defined in relation to some linear dimension and that characterizes the range of forces between particles, where $\lambda$ is the de Broglie wavelength of the particle and $a$ is the solid target particle radius and that $\phi \gg \lambda / a$, which means that the deflection of the incident trajectory must be well defined. The two criteria are equivalent to $E_{1} \gg E_{1}^{*}$ and to $\phi^{2} E_{1} \gg E_{1}^{*}$, respectively, and since $\phi$ is always less than unity, or in the worst case, of the same order as unity, the latter is sufficient for both, that is:

$$
\phi^{2} E_{1} \gg E_{1}^{*}, \quad \text { with } \quad E_{1}^{*}=4 E_{\mathrm{R}} \frac{m_{0}}{M_{1}}\left(\frac{a_{0}}{a}\right)^{2},
$$

where $a_{0}$ is the Bohr radius and refers to our scale of distance, $E_{\mathrm{R}}$ is the Rydberg energy and refers to our scale of energy, and $m_{0}$ is the mass of the electron. For a more detailed 
account of these criteria and an introduction to the problematic of interaction forces refer to, for instance, Thompson (1969). At this point we shall introduce the concept of differential scattering cross-section, $\mathrm{d} \sigma$, defined for scattering by an atom into a differential solid angle, $\mathrm{d} \omega$, where $^{2}$

$$
\mathrm{d} \omega=2 \pi \sin \phi \mathrm{d} \phi .
$$

The total number of primary collisions per unit volume, $N$, with scattering angle $>\phi$ is:

$$
N=N_{0} \Phi \sigma_{\mathrm{p}}
$$

where $N_{0}$ is the atomic density of the solid (or the number of atoms per unit volume in the solid), $\Phi$ is the fluence (or integrated flux) and $\sigma_{\mathrm{p}}$ is the total scattering cross-section for this restricted class of primary collisions defined by:

$$
\sigma_{\mathrm{p}}=2 \pi \int_{\phi}^{\pi} \frac{\mathrm{d} \sigma}{\mathrm{d} \omega} \sin \phi \mathrm{d} \phi .
$$

The concentration of displaced atoms (or displacement dose), $C_{\mathrm{d}}$, due to primary collisions is simply:

$$
C_{\mathrm{d}}=\frac{N}{N_{0}} \quad \text { or } \quad C_{\mathrm{d}}=\Phi \sigma_{\mathrm{p}} .
$$

An alternative formulation to the above set of four equations is obtained by differentiating equation (8), rearranging the trigonometric terms, solving for $\sin \phi \mathrm{d} \phi$, and using equation (14) in order to obtain the following new set of equations where the recoil angle $\phi$ no longer explicitly appears, namely, the differential solid angle $\mathrm{d} \omega$ :

$$
\mathrm{d} \omega=\frac{4 \pi}{\Lambda E_{1}} \mathrm{~d} E_{2}
$$

The total number of primary collisions per unit volume, $N$, with recoil energy $\leqslant \hat{E}_{2}$ or $\Lambda E_{1}$ (and obviously $>\check{E}_{2}$ ) is identical to equation (15), where the total scattering cross-section for this restricted class of primary collisions, $\sigma_{\mathrm{p}}$, is now defined by:

$$
\sigma_{\mathrm{p}}=\int_{\check{E}_{2}}^{\Lambda E_{1}} \frac{\mathrm{d} \sigma}{\mathrm{d} E_{2}} \mathrm{~d} E_{2},
$$

and the concentration of displaced atoms (or displacement dose), $C_{\mathrm{d}}$, due to primary collisions is also identical to equation (17), whereas the energy spectrum of primary collisions between incident particles and atoms in the solid, $C_{\mathrm{d}}^{\prime}$, is the fingerprint of the displacement process and is evaluated as follows:

$$
C_{\mathrm{d}}^{\prime}=\Phi \frac{\mathrm{d} \sigma}{\mathrm{d} E_{2}}
$$

The probability, $P\left(E_{2}\right) \mathrm{d} E_{2}$, of primary recoil in $\mathrm{d} E_{2}$ at $E_{2}$ is clearly the ratio of the differential and total scattering cross-sections, namely:

$$
P\left(E_{2}\right) \mathrm{d} E_{2}=\frac{1}{\sigma_{\mathrm{p}}} \frac{\mathrm{d} \sigma}{\mathrm{d} E_{2}} \mathrm{~d} E_{2},
$$

and therefore the fraction (or cumulative probability) of primary recoils, $f\left(E_{2}\right)$, with energy $\leqslant E_{2}$ over the entire range of interest from $\check{E}_{2}$ to $\Lambda E_{1}$ is simply obtained by integrating equation (21), as follows:

$$
f\left(E_{2}\right)=\frac{1}{\sigma_{\mathrm{p}}} \int_{\check{E}_{2}}^{E_{2}} \frac{\mathrm{d} \sigma}{\mathrm{d} E_{2}} \mathrm{~d} E_{2},
$$

2 A more accurate definition of differential scattering cross-section emerges from the general theory of collision orbits described in, for instance, Thompson (1969). 
thus the average primary recoil energy over the entire range of interest from $\check{E}_{2}$ to $\Lambda E_{1}, \bar{E}_{2}$, is defined by:

$$
\bar{E}_{2}=\frac{1}{\sigma_{\mathrm{p}}} \int_{\check{E}_{2}}^{\Lambda E_{1}} E_{2} \frac{\mathrm{d} \sigma}{\mathrm{d} E_{2}} \mathrm{~d} E_{2} .
$$

For the evaluation of $C_{\mathrm{d}}, C_{\mathrm{d}}^{\prime}, f\left(E_{2}\right)$ and $\bar{E}_{2}$, the appropriate differential scattering crosssections are needed and will be introduced $a d$ hoc in the next subsections according to the type of incident particle, i.e., neutrons and positive ions.

\subsection{Monoenergetic neutrons}

The simplest case of all presents itself when the incident particles are monoenergetic neutrons (below ca $2 \mathrm{MeV}$ ), since the probability of scattering per unit solid angle is nearly independent of scattering angle and therefore the differential scattering cross-section is essentially isotropic (in the $\mathcal{G}$ system), and the collisions resemble those between elastic hard spheres (Thompson 1969). The neutrons emitted during the fission of a ${ }^{235} \mathrm{U}$ atom are distributed in energy from about 0.5 to $10 \mathrm{MeV}$. Since the average of the distribution is located at about $1.5 \mathrm{MeV}$, we shall use this value as being characteristic of fission neutrons in this discussion (Billington and Crawford 1961). Thus, the approximate expression for the differential scattering cross-section in the case of monoenergetic neutrons is:

$$
\mathrm{d} \sigma=\frac{\sigma_{\mathrm{e}}}{4 \pi} \mathrm{d} \omega \quad \text { or } \quad \mathrm{d} \sigma=\frac{\sigma_{\mathrm{e}}}{\Lambda E_{1}} \mathrm{~d} E_{2},
$$

where $\sigma_{\mathrm{e}}$ is the total elastic scattering cross-section (of the order of 1 to 4 barn). The total scattering cross-section for this restricted class of primary collisions, $\sigma_{\mathrm{p}}$, is readily obtained integrating equation (24) from $\check{E}_{2}$ to $\Lambda E_{1}$, yielding:

$$
\sigma_{\mathrm{p}}=\sigma_{\mathrm{e}}\left(1-\frac{\check{E}_{2}}{\Lambda E_{1}}\right),
$$

and the concentration of displaced atoms (or displacement dose), $C_{\mathrm{d}}$, due to primary collisions with monoenergetic neutrons is simply:

$$
C_{\mathrm{d}}=\Phi \sigma_{\mathrm{e}}\left(1-\frac{\check{E}_{2}}{\Lambda E_{1}}\right),
$$

as depicted in figure 4(a), whereas the energy spectrum of primary collisions between incident particles and atoms in the solid, $C_{\mathrm{d}}^{\prime}$, is the fingerprint of the displacement process and is evaluated by differentiating equation (26) and explicitly using the integral of the differential scattering cross-section as defined in equation (24) instead of equation (25), for the latter does not depend explicitly on $E_{2}$, as follows:

$$
C_{\mathrm{d}}^{\prime}=\frac{\mathrm{d} C_{\mathrm{d}}}{\mathrm{d} E_{2}}=\Phi \frac{\sigma_{\mathrm{e}}}{\Lambda E_{1}}
$$

as shown in figure $4(\mathrm{~b})$. The fraction of primary recoils, $f\left(E_{2}\right)$, with energy $\leqslant E_{2}$ over the entire range of interest from $\breve{E}_{2}$ to $\Lambda E_{1}$, is similarly obtained integrating equation (24) from $\check{E}_{2}$ to $E_{2}$ (instead of $\left.\Lambda E_{1}\right)$ as follows:

$$
f\left(E_{2}\right)=\frac{E_{2}-\check{E}_{2}}{\Lambda E_{1}-\check{E}_{2}},
$$

as depicted in figure 4(c). Then, the average primary recoil energy over the entire range of interest from $\check{E}_{2}$ to $\Lambda E_{1}, \bar{E}_{2}$, is defined by:

$$
\bar{E}_{2}=\frac{1}{2}\left(\Lambda E_{1}+\check{E}_{2}\right) \text {, }
$$




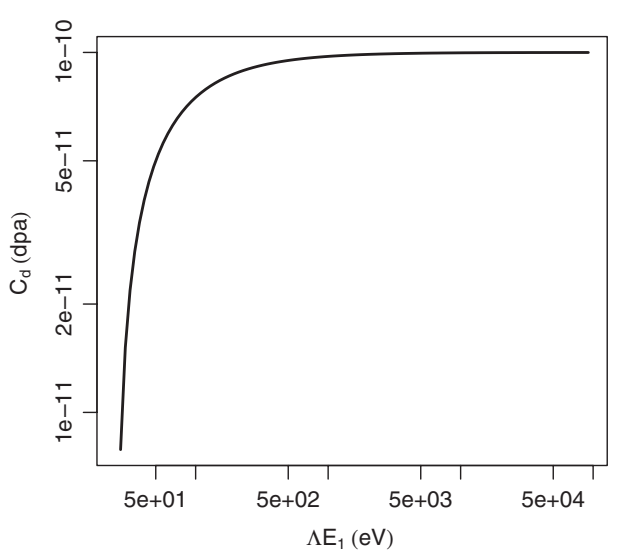

(a)

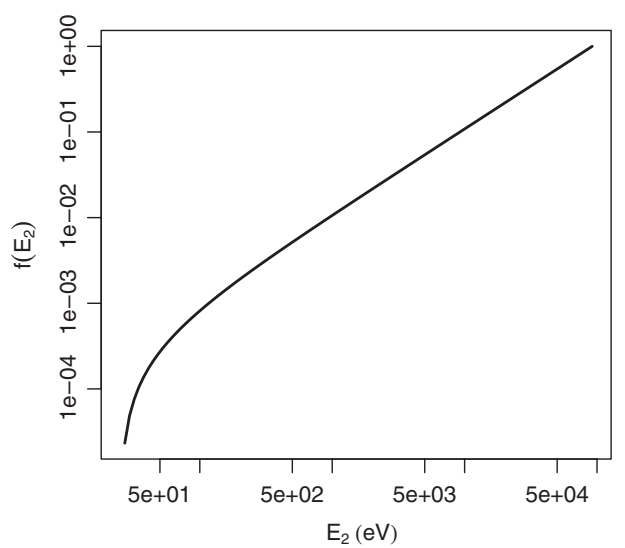

(c)

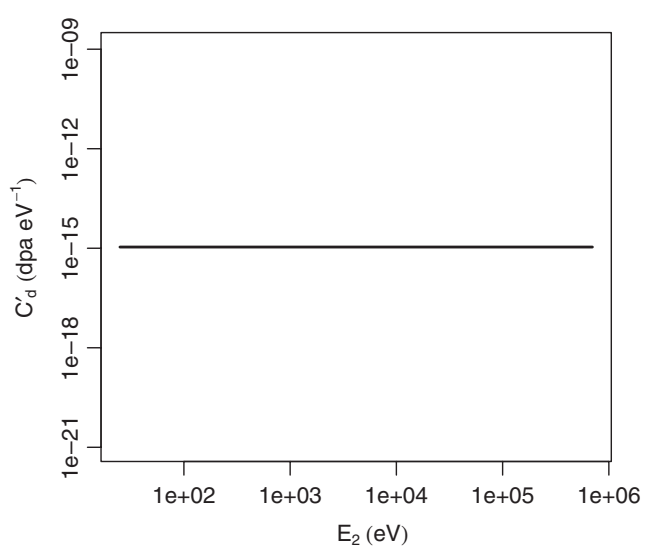

(b)

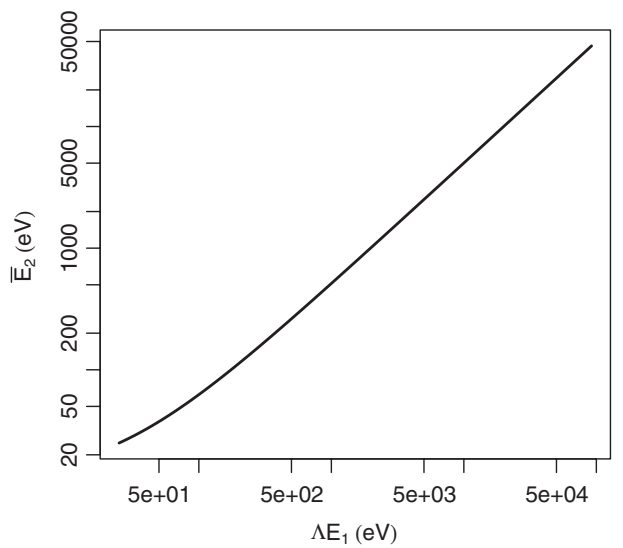

(d)

Figure 4. Damage distributions for $1.5 \mathrm{MeV}$ neutrons (maximum) at a fluence of $10^{14} \mathrm{~cm}^{-2}$ in pure copper, namely (a) concentration of displaced atoms (or displacement dose), $C_{\mathrm{d}}$, due to primary collisions with monoenergetic neutrons, (b) energy spectrum of primary collisions between incident particles and atoms in the solid, $C_{\mathrm{d}}^{\prime}$, (c) fraction of primary recoils, $f\left(E_{2}\right)$, with energy $\leqslant E_{2}$ over the entire range from $\check{E}_{2}$ to $\Lambda E_{1}$, and (d) average primary recoil energy, $\bar{E}_{2}$, over the entire range from $\check{E}_{2}$ to $\Lambda E_{1}$.

as shown in figure 4(d). The above remarks apply to fission neutrons only. For a more detailed analysis of the case of reactor spectrum neutrons in which the fission spectrum is degraded by a moderator refer to, for instance, Thompson (1969), p 126, whereas for a brief analysis refer to, for instance, Billington and Crawford (1961), p 18.

\subsection{Positive ions}

It is well known that the solution of the collision orbits problem for a simple Coulomb potential leads to the Rutherford scattering law $\mathrm{d} \sigma \sim \operatorname{cosec}^{4}(\phi / 2) \mathrm{d} \omega$ (see, for instance, Thompson (1969)). Thus, for this particular potential, the expression for the differential scattering cross-section in the case of positive ions is:

$$
\mathrm{d} \sigma=\frac{a_{0}^{2} Z_{1}^{2} Z_{2}^{2} E_{\mathrm{R}}^{2}}{\mu^{2} u_{1}^{4}} \frac{\mathrm{d} \omega}{\sin ^{4}(\phi / 2)},
$$


where $Z_{1}$ and $Z_{2}$ are the atomic numbers of the incident particle and the target atoms, $\mu=M_{1} M_{2}\left(M_{1}+M_{2}\right)^{-1}$ is the reduced mass and $u_{1}$ is the magnitude of the relative velocity of the two particles in the $\mathcal{G}$ system, $\boldsymbol{u}_{1}=\boldsymbol{U}_{1}-\left(-\boldsymbol{u}_{g}\right)=\boldsymbol{U}_{1}+\boldsymbol{u}_{g}$. If we now expand the reduced mass $\mu$ into its components $M_{1}$ and $M_{2}$ and further note that the term $M_{1}^{2} u_{1}^{4}$ is simply $4 E_{1}^{2}$ some useful simplification is brought about, namely:

$$
\mathrm{d} \sigma=\frac{a_{0}^{2} Z_{1}^{2} Z_{2}^{2} E_{\mathrm{R}}^{2}\left(M_{1}+M_{2}\right)^{2}}{4 M_{2}^{2} E_{1}^{2}} \frac{\mathrm{d} \omega}{\sin ^{4}(\phi / 2)},
$$

which we now have to express as a function of the primary recoil energy, $E_{2}$, using for that particular purpose the relationships outlined in equations (8) and (18), as follows:

$$
\mathrm{d} \sigma=\frac{4 \pi a_{0}^{2} M_{1} Z_{1}^{2} Z_{2}^{2} E_{\mathrm{R}}^{2}}{M_{2} E_{1}} \frac{\mathrm{d} E_{2}}{E_{2}^{2}},
$$

hence the total scattering cross-section for this restricted class of primary collisions, $\sigma_{\mathrm{p}}$, is readily obtained integrating equation (32) from $\check{E}_{2}$ to $\Lambda E_{1}$, yielding:

$$
\sigma_{\mathrm{p}}=\frac{4 \pi a_{0}^{2} M_{1} Z_{1}^{2} Z_{2}^{2} E_{\mathrm{R}}^{2}}{M_{2} E_{1} \check{E}_{2}}\left(1-\frac{\check{E}_{2}}{\Lambda E_{1}}\right),
$$

and the concentration of displaced atoms (or displacement dose), $C_{\mathrm{d}}$, due to primary collisions with positive ions is simply:

$$
C_{\mathrm{d}}=\frac{4 \pi \Phi a_{0}^{2} M_{1} Z_{1}^{2} Z_{2}^{2} E_{\mathrm{R}}^{2}}{M_{2} E_{1} \check{E}_{2}}\left(1-\frac{\check{E}_{2}}{\Lambda E_{1}}\right),
$$

as depicted in figure 5(a), whereas the energy spectrum of primary collisions between incident particles and atoms in the solid, $C_{\mathrm{d}}^{\prime}$, is the fingerprint of the displacement process and is evaluated using for that purpose equations (20) and (32) as follows:

$$
C_{\mathrm{d}}^{\prime}=\frac{4 \pi \Phi a_{0}^{2} M_{1} Z_{1}^{2} Z_{2}^{2} E_{\mathrm{R}}^{2}}{M_{2} E_{1}} \frac{1}{E_{2}^{2}},
$$

as shown in figure $5(\mathrm{~b})$. The fraction of primary recoils, $f\left(E_{2}\right)$, with energy $\leqslant E_{2}$ over the entire range of interest from $\check{E}_{2}$ to $\Lambda E_{1}$ is similarly obtained integrating equation (32) from $\check{E}_{2}$ to $E_{2}\left(\right.$ instead of $\left.\Lambda E_{1}\right)$ as follows:

$$
f\left(E_{2}\right)=\left(1-\frac{\check{E}_{2}}{E_{2}}\right)\left(1-\frac{\check{E}_{2}}{\Lambda E_{1}}\right)^{-1},
$$

as depicted in figure 5(c). Then, the average primary recoil energy over the entire range of interest from $\check{E}_{2}$ to $\Lambda E_{1}, \bar{E}_{2}$, is defined by:

$$
\bar{E}_{2}=\check{E}_{2} \ln \left(\frac{\Lambda E_{1}}{\check{E}_{2}}\right)\left(1-\frac{\check{E}_{2}}{\Lambda E_{1}}\right)^{-1},
$$

as shown in figure $5(\mathrm{~d})$.

\section{Analysis of the collision cascade}

In this section we will be mainly concerned with the total production of displaced atoms by both primary and secondary recoils and therefore we aim at the determination of the damage function $v\left(E_{2}\right)$ which yields the average number of displaced atoms per primary knock-on atom, including the primaries themselves, due to a recoil at $E_{2}$. Clearly, at an energy of $\approx 2 \check{E}_{2}$ 


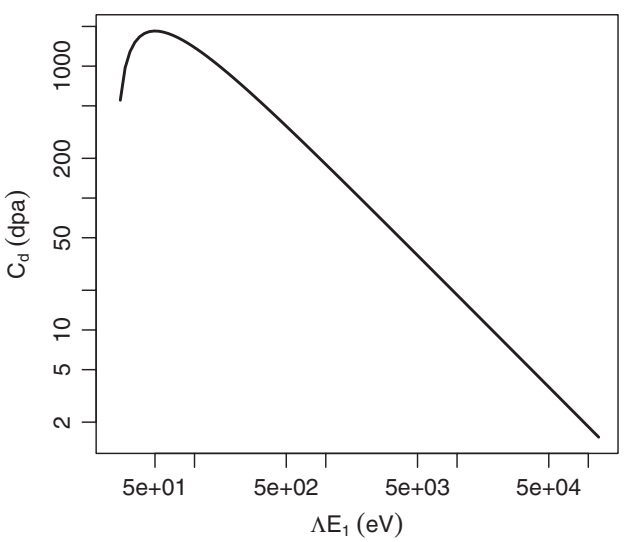

(a)

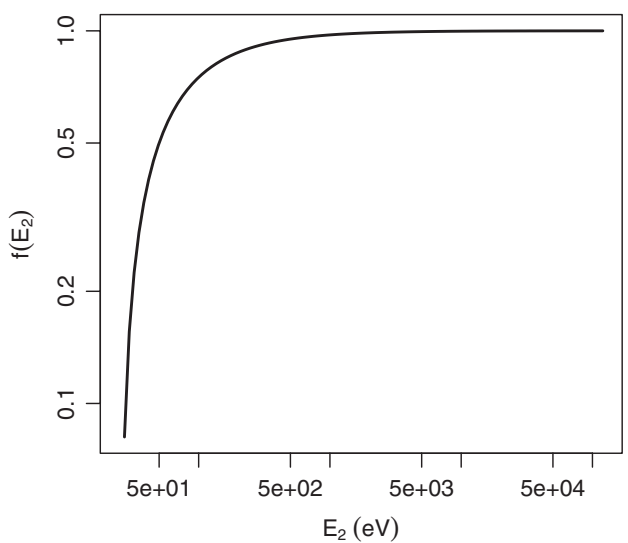

(c)

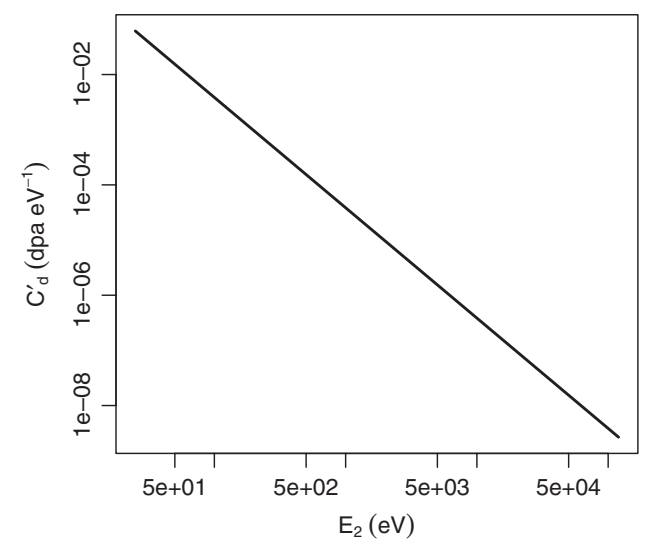

(b)

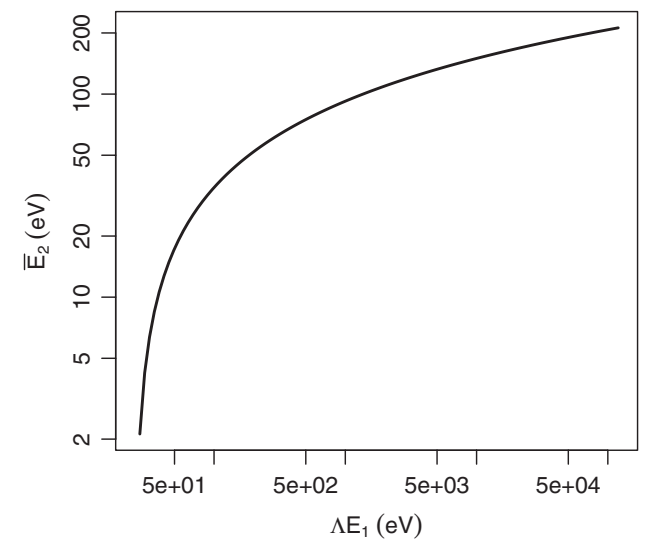

(d)

Figure 5. Damage distributions for $120 \mathrm{keV}$ positive self-ions (maximum) at a fluence of $10^{14} \mathrm{~cm}^{-2}$ in pure copper, namely, (a) concentration of displaced atoms (or displacement dose), $C_{\mathrm{d}}$, due to primary collisions with positive ions, (b) energy spectrum of primary collisions between incident particles and atoms in the solid, $C_{\mathrm{d}}^{\prime}$, (c) fraction of primary recoils, $f\left(E_{2}\right)$, with energy $\leqslant E_{2}$ over the entire range from $\check{E}_{2}$ to $\Lambda E_{1}$, and (d) average primary recoil energy, $\bar{E}_{2}$, over the entire range from $\breve{E}_{2}$ to $\Lambda E_{1}$.

it becomes possible to create two Frenkel pairs (i.e., two vacancies + two interstitials) as a result of a single primary event. The second event results thus from an atom-atom collision and at still higher energies, tertiary and high-order progeny may be created and produce a displacement cascade.

Calculations of $v\left(E_{2}\right)$ have been performed subject to a series of assumptions and constraints, e.g., it is assumed that the interaction between atoms is hard-sphere in nature and that all energy losses for a recoil are disregarded, insofar as they contribute to atomic displacements, until the energy has been degraded by collisions with electrons in the solid to a value below a critical energy $E_{\text {ex }}$, namely:

$$
E_{\mathrm{ex}} \approx \frac{M_{2} E_{\mathrm{i}}^{2}}{4 m_{0} E_{\mathrm{R}}}
$$

where $E_{\mathrm{i}}$ is the first ionization energy of the solid. Also, when $E_{2}<E_{\mathrm{ex}}$, all further energy degradation is assumed to result only from elastic atom-atom collisions. Finally, an atom 
is assumed to always be ejected from its lattice site position if it receives an energy transfer $E_{2}>\breve{E}_{2}$ but to remain in the region of the atom-atom collision if it fails to receive this amount of energy (see, for instance, Sosin and Bauer (1969), or Thompson (1969), for a more stringent set of assumptions and constraints).

A preliminary formulation of $v\left(E_{2}\right)$ based on a very much simplified model, first used by Kinchin and Pease (1955), required that each atom possess a kinetic energy $E_{2}>\breve{E}_{2}$ after the collision for both to be displaced, and allowed for neither to be displaced if neither atom leaves the collision with an energy $E_{2}<\check{E}_{2}$. When the primary atom leaves the collision with an energy $E_{2}<\check{E}_{2}$ but the secondary emerges with an energy $E_{2}>\check{E}_{2}$, the primary atom is assumed to occupy the original site of the secondary atom. Under this specific scheme, Kinchin and Pease (1955) focused attention on replacement collisions which contribute no additional atomic displacement in a pure monoatomic metal, but do play a crucial role in ordered alloys.

On the basis of the assumptions made in the Kinchin and Pease (1955) formulation an approximate value for $v\left(E_{2}\right)$ can be obtained by a simple argument. In a truly hard-sphere collision, each of the two atoms has identical probability of emerging from the collision with a specific recoil energy $E_{2}$, and therefore the energy is shared equally between the two atoms, on average. If we extend this rationale to subsequent collisions it is clear that the energy is distributed equally among all the atoms in the cascade until $E_{2}<2 \check{E}_{2}$. At this specific instant no further multiple displacements can occur. Accordingly, the total number of atomic displacements produced in the solid during the cascade, $v\left(E_{2}\right)$, is simply formulated as follows (see, for instance, Chadderton (1965) and Norgett et al (1975) for a more detailed account of the various damage function mathematical forms and of the displacement efficiency, respectively):

$$
v\left(E_{2}\right)=\left\{\begin{array}{lll}
0 & ; & E_{2}<\check{E}_{2} \\
1 & ; & \check{E}_{2}<E_{2}<2 \check{E}_{2} \\
E_{2} / 2 \check{E}_{2} & ; & 2 \breve{E}_{2}<E_{2}<E_{\mathrm{ex}} \\
E_{\mathrm{ex}} / 2 \check{E}_{2} & ; & E_{2}>E_{\mathrm{ex}},
\end{array}\right.
$$

as depicted in figures $6(\mathrm{a})$ and (b).

In order to incorporate multiple displacements into cross-section calculations, or in other words, to evaluate the displacement cross-section, the damage function (or multiplicity factor), $v\left(E_{2}\right)$, is ordinarily introduced in the integrand on the right-hand side of equation (19), namely:

$$
\sigma_{\mathrm{d}}=\int_{\check{E}_{2}}^{\Lambda E_{1}} v\left(E_{2}\right) \frac{\mathrm{d} \sigma}{\mathrm{d} E_{2}} \mathrm{~d} E_{2},
$$

where the integral can be evaluated using for that purpose the definition of the average number of (multiple) atomic displacements, $\bar{v}\left(\Lambda E_{1}\right)$, over the entire energy spectrum of primary knock-on atoms from $\check{E}_{2}$ to $\Lambda E_{1}$ as follows:

$$
\bar{v}\left(\Lambda E_{1}\right)=\int_{\check{E}_{2}}^{\Lambda E_{1}} v\left(E_{2}\right) \frac{\mathrm{d} \sigma}{\mathrm{d} E_{2}} \mathrm{~d} E_{2}\left(\int_{\check{E}_{2}}^{\Lambda E_{1}} \frac{\mathrm{d} \sigma}{\mathrm{d} E_{2}} \mathrm{~d} E_{2}\right)^{-1},
$$

and hence

$$
\sigma_{\mathrm{d}}=\sigma_{\mathrm{p}} \bar{v}\left(\Lambda E_{1}\right) .
$$

The concentration of displaced atoms (or displacement dose), $C_{\mathrm{d}}$, due to secondary collisions is then:

$$
C_{\mathrm{d}}=\Phi \sigma_{\mathrm{p}} \bar{v}\left(\Lambda E_{1}\right)
$$




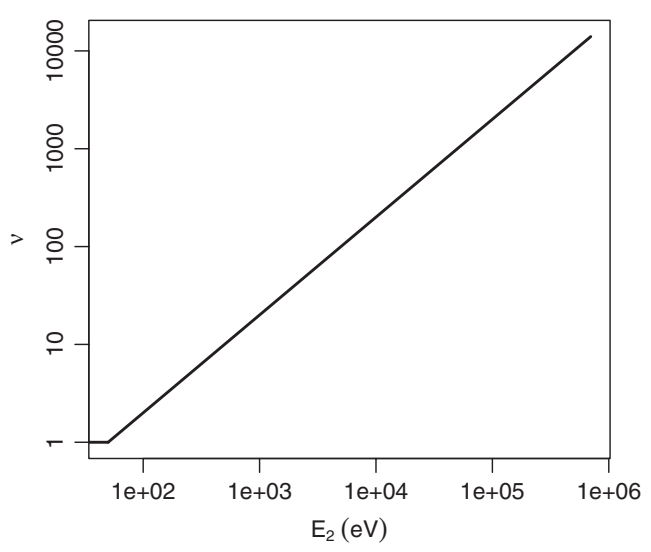

(a)

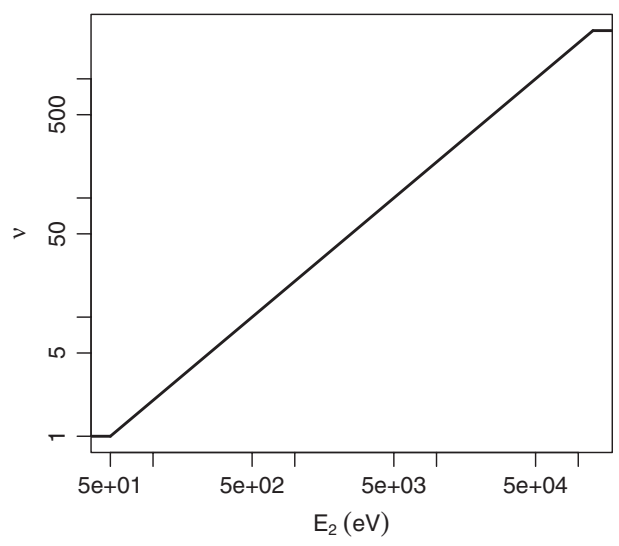

(b)

Figure 6. Example of damage functions, $v\left(E_{2}\right)$, according to the Kinchin and Pease (1955) formulation, for $700 \mathrm{keV}$ positive self-ions (maximum) in pure copper, namely, (a) ignoring the effect of the electronic excitation in the solid, and (b) taking into account the electronic excitation in the solid, $E_{\mathrm{ex}} \approx 127 \mathrm{keV}$, over the entire range from $\check{E}_{2}$ to $\Lambda E_{1}$ (with saturation of atomic displacement production above $\left.E_{\mathrm{ex}}\right)$.

whereas the energy spectrum of secondary collisions in the solid, $C_{\mathrm{d}}^{\prime}$, is simply:

$$
C_{\mathrm{d}}^{\prime}=\Phi v\left(E_{2}\right) \frac{\mathrm{d} \sigma}{\mathrm{d} E_{2}}
$$

The probability, $P\left(E_{2}\right) \mathrm{d} E_{2}$, of secondary recoil in $\mathrm{d} E_{2}$ at $E_{2}$ is the ratio of the differential and the total scattering cross-sections, namely:

$$
P\left(E_{2}\right) \mathrm{d} E_{2}=\frac{v\left(E_{2}\right)}{\sigma_{\mathrm{p}} \bar{v}\left(\Lambda E_{1}\right)} \frac{\mathrm{d} \sigma}{\mathrm{d} E_{2}} \mathrm{~d} E_{2},
$$

and therefore the fraction (or cumulative probability) of secondary recoils, $f\left(E_{2}\right)$, with energy $\leqslant E_{2}$ over the entire range of interest from $\check{E}_{2}$ to $\Lambda E_{1}$ is simply obtained as follows:

$$
f\left(E_{2}\right)=\frac{1}{\sigma_{\mathrm{p}} \bar{v}\left(\Lambda E_{1}\right)} \int_{\check{E}_{2}}^{E_{2}} v\left(E_{2}\right) \frac{\mathrm{d} \sigma}{\mathrm{d} E_{2}} \mathrm{~d} E_{2},
$$

thus the average secondary recoil energy over the entire range of interest from $\check{E}_{2}$ to $\Lambda E_{1}, \bar{E}_{2}$, is defined by:

$$
\bar{E}_{2}=\frac{1}{\sigma_{\mathrm{p}} \bar{v}\left(\Lambda E_{1}\right)} \int_{\check{E}_{2}}^{\Lambda E_{1}} v\left(E_{2}\right) E_{2} \frac{\mathrm{d} \sigma}{\mathrm{d} E_{2}} \mathrm{~d} E_{2} .
$$

\subsection{Monoenergetic neutrons}

A complete accounting of the total number of atomic displacements triggered by fission neutrons in a solid is, again, the simplest case of all in the frame of cascade analysis and we will build a new set of results which rely directly on the material introduced previously. In this regard, the average damage function, $\bar{v}\left(\Lambda E_{1}\right)$, is simply obtained by integrating equation (41) as follows:

$$
\bar{v}\left(\Lambda E_{1}\right)=\frac{1}{\sigma_{\mathrm{p}}}\left(\int_{\check{E}_{2}}^{2 \check{E}_{2}} \frac{\mathrm{d} \sigma}{\mathrm{d} E_{2}} \mathrm{~d} E_{2}+\frac{1}{2 \check{E}_{2}} \int_{2 \check{E}_{2}}^{\Lambda E_{1}} E_{2} \frac{\mathrm{d} \sigma}{\mathrm{d} E_{2}} \mathrm{~d} E_{2}\right),
$$




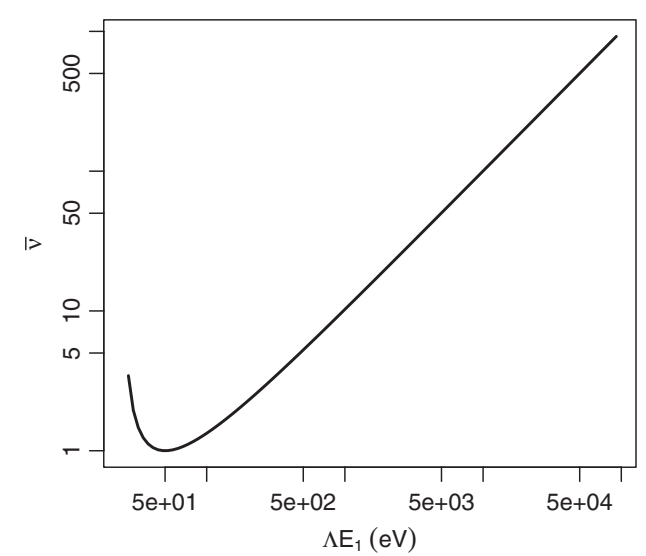

Figure 7. Average damage function, $\bar{v}\left(\Lambda E_{1}\right)$, for $1.5 \mathrm{MeV}$ neutrons (maximum) in pure copper, over the entire range from $\check{E}_{2}$ to $\Lambda E_{1}$ (electronic excitation in the solid due to ionization does not play a role, for $\left.\Lambda E_{1}<E_{\mathrm{ex}}\right)$.

and hence

$$
\bar{v}\left(\Lambda E_{1}\right)=\frac{\Lambda E_{1}}{4 \check{E}_{2}}\left(1-\frac{\check{E}_{2}}{\Lambda E_{1}}\right)^{-1},
$$

as shown in figure 7 , whereas the displacement cross-section, $\sigma_{\mathrm{d}}$, is then defined by

$$
\sigma_{\mathrm{d}}=\sigma_{\mathrm{e}} \frac{\Lambda E_{1}}{4 \check{E}_{2}} .
$$

The concentration of displaced atoms (or displacement dose), $C_{\mathrm{d}}$, due to secondary collisions is simply:

$$
C_{\mathrm{d}}=\Phi \sigma_{\mathrm{e}} \frac{\Lambda E_{1}}{4 \check{E}_{2}}
$$

as depicted in figure 8(a), and the energy spectrum of secondary collisions in the solid, $C_{\mathrm{d}}^{\prime}$, is the fingerprint of the complete displacement process and is defined by:

$$
C_{\mathrm{d}}^{\prime}=\Phi \frac{\sigma_{\mathrm{e}}}{\Lambda E_{1}} \frac{E_{2}}{2 \check{E}_{2}}
$$

as shown in figure 8(b). The fraction of secondary recoils, $f\left(E_{2}\right)$, with energy $\leqslant E_{2}$ over the entire range of interest from $\check{E}_{2}$ to $\Lambda E_{1}$ is:

$$
f\left(E_{2}\right)=\left(\frac{E_{2}}{\Lambda E_{1}}\right)^{2},
$$

as depicted in figure $8(\mathrm{c})$. Then, the average secondary recoil energy over the entire range of interest from $\check{E}_{2}$ to $\Lambda E_{1}$ is defined by:

$$
\bar{E}_{2}=\frac{2}{3}\left(\frac{\check{E}_{2}^{3}+\Lambda^{3} E_{1}^{3}}{\Lambda^{2} E_{1}^{2}}\right),
$$

as shown in figure $8(\mathrm{~d})$. 


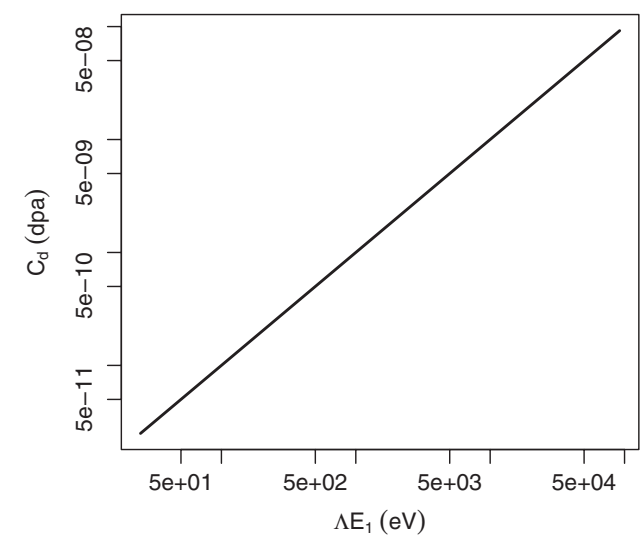

(a)

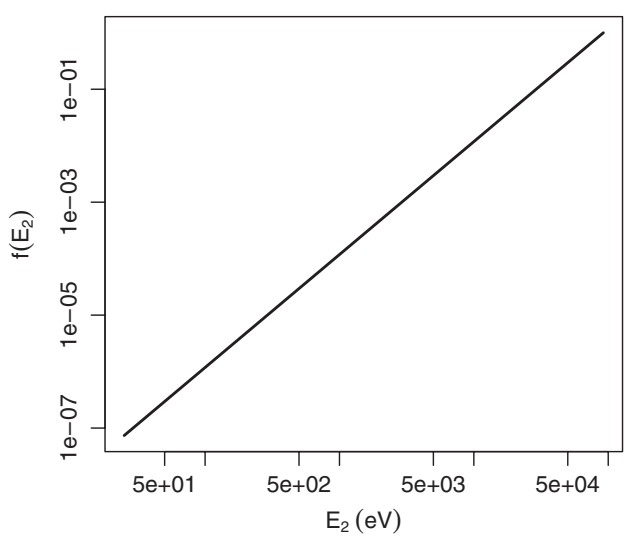

(c)

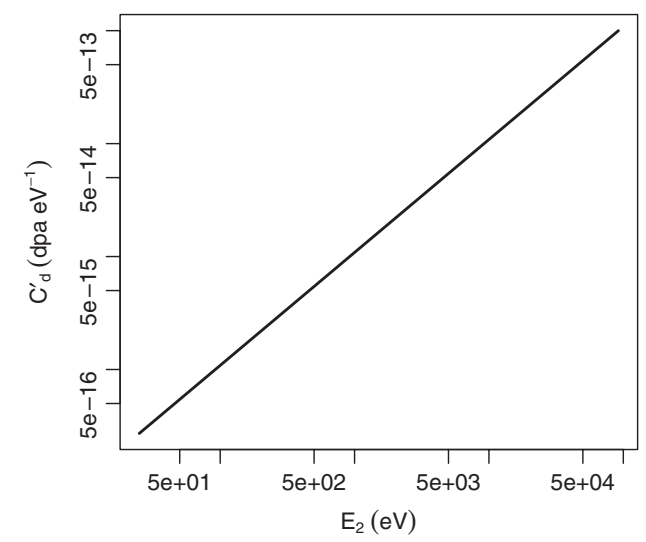

(b)

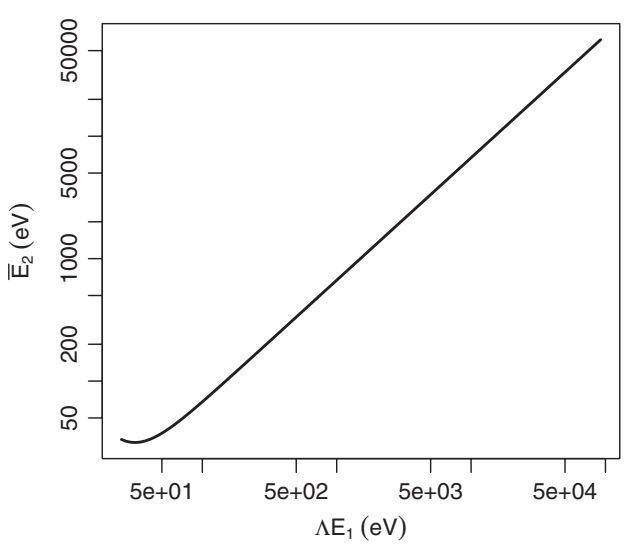

(d)

Figure 8. Damage distributions for $1.5 \mathrm{MeV}$ neutrons (maximum) at a fluence of $10^{14} \mathrm{~cm}^{-2}$ in pure copper, namely, (a) concentration of displaced atoms (or displacement dose), $C_{\mathrm{d}}$, due to secondary collisions, (b) energy spectrum of secondary collisions in the solid, $C_{\mathrm{d}}^{\prime}$, (c) fraction of secondary recoils, $f\left(E_{2}\right)$, with energy $\leqslant E_{2}$ over the entire range from $\check{E}_{2}$ to $\Lambda E_{1}$, and (d) average secondary recoil energy, $\bar{E}_{2}$, over the entire range from $\check{E}_{2}$ to $\Lambda E_{1}$.

\subsection{Positive ions}

A complete accounting of the total number of atomic displacements triggered by positive ions in a solid requires a complete analysis of the cascade and therefore we will build a new set of results which are directly based on the material previously introduced. In this framework, the average damage function, $\bar{v}\left(\Lambda E_{1}\right)$, is thus obtained:

$$
\bar{v}\left(\Lambda E_{1}\right)=\frac{1}{2}\left[1+\ln \left(\frac{\Lambda E_{1}}{2 \check{E}_{2}}\right)\right]\left(1-\frac{\check{E}_{2}}{\Lambda E_{1}}\right)^{-1},
$$

as shown in figure 9 , whereas the displacement cross-section, $\sigma_{\mathrm{d}}$, is then defined by

$$
\sigma_{\mathrm{d}}=\frac{2 \pi a_{0}^{2} M_{1} Z_{1}^{2} Z_{2}^{2} E_{\mathrm{R}}^{2}}{M_{2} E_{1} \check{E}_{2}}\left[1+\ln \left(\frac{\Lambda E_{1}}{2 \check{E}_{2}}\right)\right] .
$$




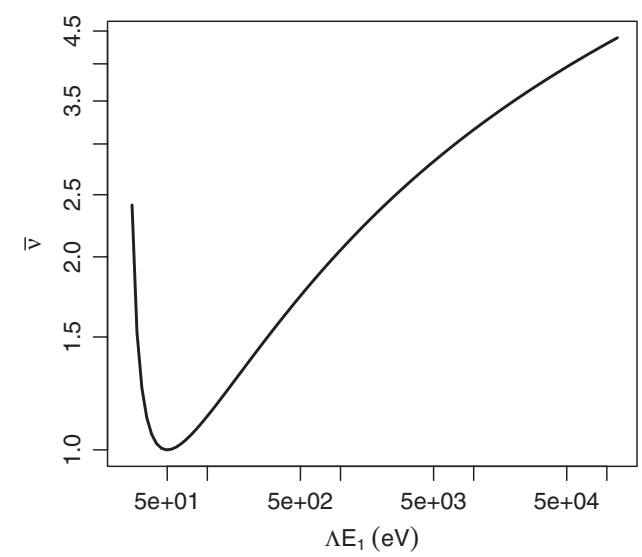

Figure 9. Average damage function, $\bar{v}\left(\Lambda E_{1}\right)$, for $120 \mathrm{keV}$ self-ions (maximum) in pure copper, over the entire range from $\check{E}_{2}$ to $\Lambda E_{1}$ (electronic excitation in the solid due to ionization does not play a role, for $\left.\Lambda E_{1}<E_{\mathrm{ex}}\right)$.

The concentration of displaced atoms (or displacement dose), $C_{\mathrm{d}}$, due to secondary collisions is simply:

$$
C_{\mathrm{d}}=\frac{2 \pi \Phi a_{0}^{2} M_{1} Z_{1}^{2} Z_{2}^{2} E_{\mathrm{R}}^{2}}{M_{2} E_{1} \check{E}_{2}}\left[1+\ln \left(\frac{\Lambda E_{1}}{2 \check{E}_{2}}\right)\right],
$$

as depicted in figure 10(a), and the energy spectrum of secondary collisions in the solid, $C_{\mathrm{d}}^{\prime}$, is the fingerprint of the complete displacement process and is defined by:

$$
C_{\mathrm{d}}^{\prime}=\frac{2 \pi \Phi a_{0}^{2} M_{1} Z_{1}^{2} Z_{2}^{2} E_{\mathrm{R}}^{2}}{M_{2} E_{1} \check{E}_{2}} \frac{1}{E_{2}},
$$

as shown in figure $10(\mathrm{~b})$. The fraction of secondary recoils, $f\left(E_{2}\right)$, with energy $\leqslant E_{2}$ over the entire range of interest from $\breve{E}_{2}$ to $\Lambda E_{1}$ is:

$$
f\left(E_{2}\right)=\left[1+\ln \left(\frac{E_{2}}{2 \check{E}_{2}}\right)\right]\left[1+\ln \left(\frac{\Lambda E_{1}}{2 \check{E}_{2}}\right)\right]^{-1},
$$

as depicted in figure $10(\mathrm{c})$. Then, the average secondary recoil energy over the entire range of interest from $\check{E}_{2}$ to $\Lambda E_{1}$ is defined by:

$$
\bar{E}_{2}=\left[2(\ln 2-1) \check{E}_{2}+\Lambda E_{1}\right]\left[1+\ln \left(\frac{\Lambda E_{1}}{2 \check{E}_{2}}\right)\right]^{-1},
$$

as shown in figure $10(d)$.

\section{Discussion}

Atomic displacement in solids, namely, the complete accounting of Frenkel pairs created right after the formation of the primary knock-on atom (or primary recoil) until no further multiple displacements can occur, is an exercise of great fundamental and technological importance and therefore an analytical treatment of this problem is not only a very fine mathematical-physics assessment with its own merit but also serves as the basis of more complex, sophisticated damage models. 


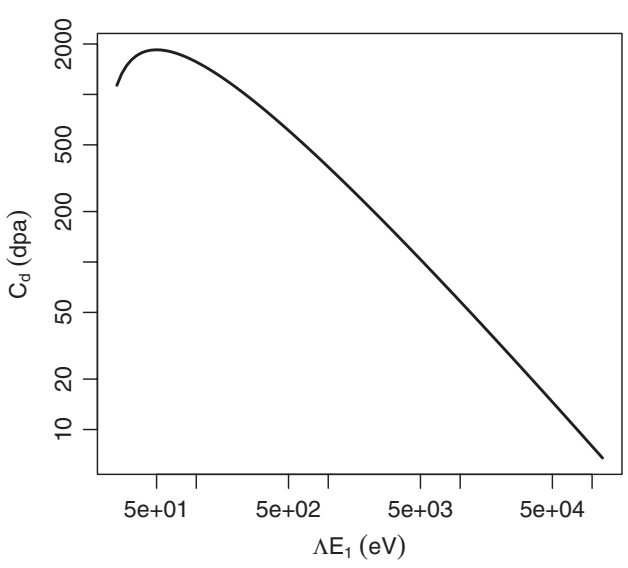

(a)

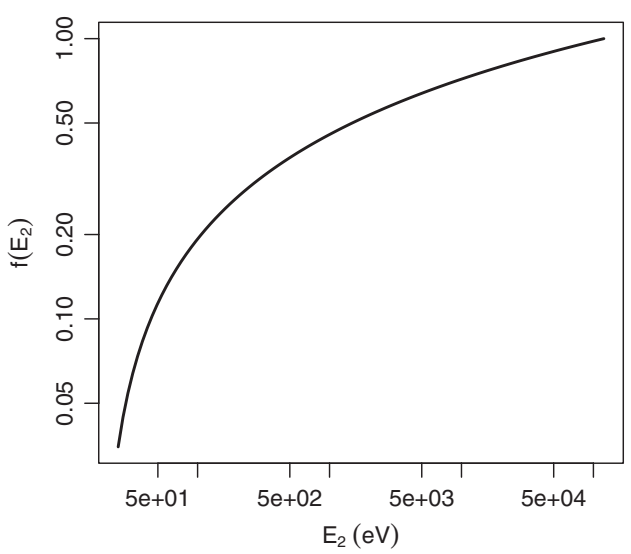

(c)

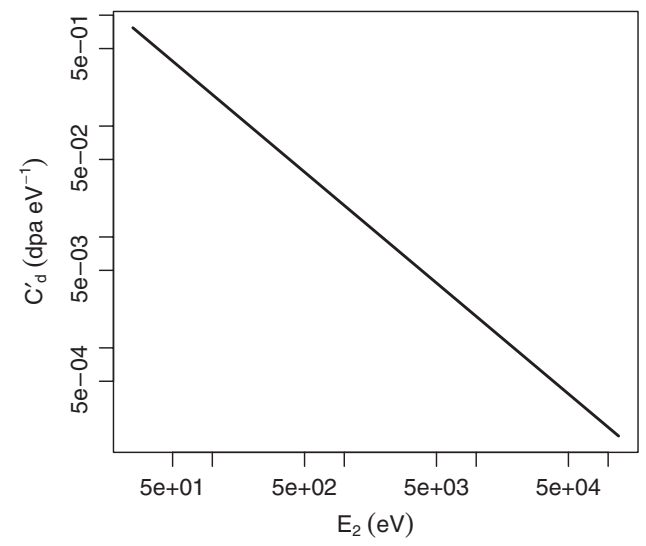

(b)

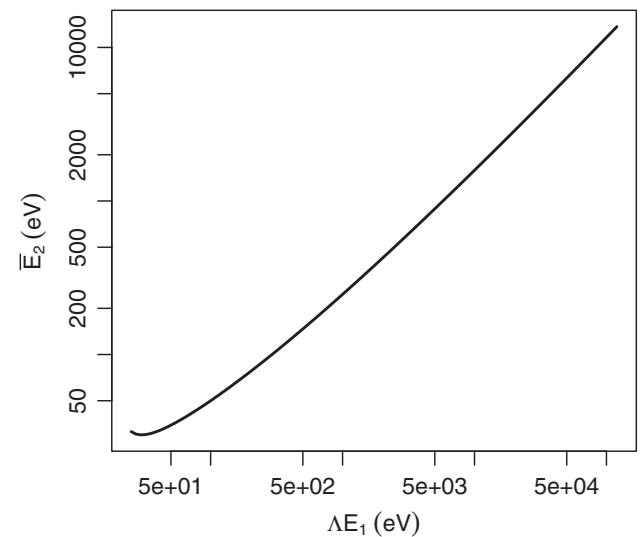

(d)

Figure 10. Damage distributions for $120 \mathrm{keV}$ self-ions (maximum) at a fluence of $10^{14}$ $\mathrm{cm}^{-2}$ in pure copper, namely, (a) concentration of displaced atoms (or displacement dose), $C_{\mathrm{d}}$, due to secondary collisions, (b) energy spectrum of secondary collisions in the solid, $C_{\mathrm{d}}^{\prime}$, (c) fraction of secondary recoils, $f\left(E_{2}\right)$, with energy $\leqslant E_{2}$ over the entire range from $\check{E}_{2}$ to $\Lambda E_{1}$, and (d) average secondary recoil energy, $\bar{E}_{2}$, over the entire range from $\check{E}_{2}$ to $\Lambda E_{1}$.

After developing the theory for both primary and secondary (and higher-order progeny) recoils for neutron and positive ion irradiation we have made a series of simplifying assumptions, for instance, a relatively naive displacement damage function formulation due to Kinchin and Pease (1955) which ignores subthreshold displacements, crystallographic orientation (Chadderton 1965, Sosin and Bauer 1969) and defect recombination within the cascade (Schilling and Ullmaier 1994). However, the most peculiar aspects of atomic displacement in solids under neutron and positive ion irradiation become evident right at the end of this mathematical-physics exercise. It is noteworthy to realize that primary and secondary (and higher-order progeny) atomic displacements follow different physical formulations for both neutron and positive ion irradiation and that despite their inherent particulars - a direct consequence of the distinct nature of the total scattering cross-sectionsthe creation of displacement damage is basically due to secondary recoils (compare, for instance, figures 4(b) and 5(b) with figures 8(b) and 10(b), respectively). 
A key concept in displacement damage theory is the concentration of displaced atoms (or displacement dose) as opposed to the more traditional concept of fluence. In fact, the consistent use of an adequate concept that correctly accounts for the atomic displacement in solids was lacking until not so long ago. The use of the physical concept of concentration of displaced atoms (or displacement dose) permits a direct comparison between different solids irradiated with either neutrons or positive ions at different fluences, for it takes into account all these elements thus yielding a fair estimate of the total displacement damage in the solid. In general, though, recombination (or annealing) will occur during (and after) irradiation being the annealing rate proportional to the square of the number of Frenkel pairs (see, for instance, Anno (1985)).

In this paper we have presented two case studies, namely, of neutron and positive ion irradiation in pure copper with $1.5 \mathrm{MeV}$ and $120 \mathrm{keV}$ primary energies, respectively, at a fluence of $10^{14} \mathrm{~cm}^{-2}$ hence illustrating the obvious differences related to the nature of the irradiation and primary energy even though the fluence is the same for both cases. Moreover, the distinct average damage functions, recoil energy spectra and average recoil energy distributions are of great physical value in the frame of the quantitative analytical models here studied and are the crux of the matter in terms of atomic displacement in solids. Further refinements to the basic theory can be derived (see, for instance, Thompson (1969)) and despite the extra gain they might bring it is sure that a more detailed picture of the whole phenomena needs also an accurate estimation of the fraction of defects which recombine (or anneal) during (and after) the irradiation using standard rate theory or, better, the production bias model (see, for instance, Golubov and Singh (2001)). The existence of several cascade damage models, all of which are in principle equally plausible, emphasizes how unreliable these calculations can be. On the basis of this fact and considering the basic assumptions common to all the models, we cannot expect such calculations to be accurate to better than a factor of perhaps 2 or 3 (Dienes and Vineyard 1957). Indeed, comparison of the analytical results with experiments indicates the need for refinement. With but a few exceptions the density of defects indicated by the experiments is smaller than the theoretical values by a factor of 3 to 10 (Billington and Crawford Jr 1961).

A rather pertinent problem in a modern treatment of atomic displacement in solids actually stems from the fact that the physical units used in almost all the classical textbooks are outdated, i.e., they refer to cgs units, or to a hybrid system of units (e.g., atomic units) instead of using the Système International (SI), introduced only in 1960. Having this specific problem in our minds we have used mainly rationalized physical expressions in which the resulting physical units are obvious. A relatively newcomer to the radiation damage community is the ratio $N / N_{0}$ or $C_{\mathrm{d}}$-which, strictly speaking, has no physical units-now commonly expressed in 'displacements per atom' or simply, dpa. In fact, this is a calculated, hypothetical measure of radiation damage and hence does not yield the residual concentration of displaced atoms actually created in the solid, but rather a measure of the number of atoms that could possibly be permanently displaced from their lattice site positions to stable interstitial sites (Heinisch et al 2002). In the same line of thought, the damage function, $v\left(E_{2}\right)$, which, again, has no physical units, might be expressed in, say, 'Frenkel pairs per primary knock-on atom' or even 'displacements per primary knock-on atom' or simply, to coin an new acronym, dppka.

Finally, the analytical formulation we derived for both neutron and positive ion irradiation is very elegant and relatively compact and therefore can be easily transcribed into a students' computational toolkit in order to quickly estimate the atomic displacement in solids. For an example of such a type of elegant mathematical-physics transcription, see, for instance, Grenander (1982) and Chaitin (1985). 


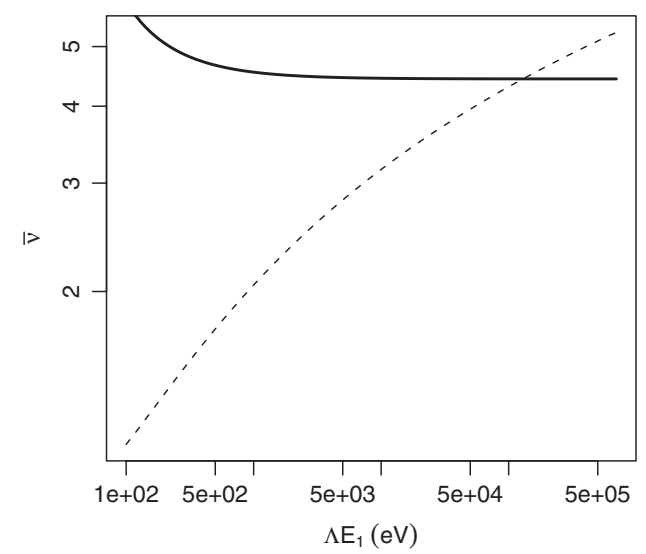

Figure 11. Average damage function, $\bar{v}\left(\Lambda E_{1}\right)$, for $700 \mathrm{keV}$ self-ions (maximum) in pure copper, either taking into account the effect of electronic excitation in the solid due to ionization (thick line) or ignoring it (thin, dashed line), over the entire range from $\check{E}_{2}$ to $\Lambda E_{1}$.

\section{Acknowledgments}

We would like to thank Dr G J Chaitin (IBM Research Division, Yorktown Heights, NY) for providing us with the reference on APL and for the inspirational role that it triggered. We would also like to kindly thank Dr M Victoria (CRPP-EPFL Fusion Technology Materials, Switzerland) for reading and commenting on the manuscript.

\section{Appendix. Analysis of the collision cascade taking into account the electronic excitation in the solid}

A correct evaluation of the average damage function, $\bar{v}\left(\Lambda E_{1}\right)$, in the $E_{2}>E_{\text {ex }}$ recoil energy limit requires complete accounting of the number of atomic displacements over three distinct regions of the damage function, $v\left(E_{2}\right)$, yielding:

$\bar{\nu}\left(\Lambda E_{1}\right)=\frac{1}{\sigma_{\mathrm{p}}}\left(\int_{\check{E}_{2}}^{2 \check{E}_{2}} \frac{\mathrm{d} \sigma}{\mathrm{d} E_{2}} \mathrm{~d} E_{2}+\frac{1}{2 \check{E}_{2}} \int_{2 \check{E}_{2}}^{E_{\mathrm{ex}}} E_{2} \frac{\mathrm{d} \sigma}{\mathrm{d} E_{2}} \mathrm{~d} E_{2}+\frac{E_{\mathrm{ex}}}{2 \check{E}_{2}} \int_{E_{\mathrm{ex}}}^{\Lambda E_{1}} \frac{\mathrm{d} \sigma}{\mathrm{d} E_{2}} \mathrm{~d} E_{2}\right)$,

and hence the derivation of all related quantities should reflect this particular result. It is clear that whenever the maximum possible energy transfer $\Lambda E_{1}$ exceeds $E_{\text {ex }}$ the effect of electronic excitation becomes important and, if completely ignored, will lead to an overestimation of the concentration of displaced atoms (or displacement dose), for instance, in the case of 1.5 MeV neutron irradiation of pure aluminium (instead of pure copper) for which $\Lambda E_{1}=$ $207 \mathrm{keV}$ and $E_{\mathrm{ex}} \approx 32 \mathrm{keV}$ or in the case of $700 \mathrm{keV}$ self-ion irradiation of pure copper for which $\Lambda E_{1}=700 \mathrm{keV}$ and $E_{\mathrm{ex}} \approx 127 \mathrm{keV}$. In either case the starting point of our mathematical analysis is identical, and therefore we will only illustrate a couple of partial results for the latter; in this regard, the average damage function, $\bar{v}\left(\Lambda E_{1}\right)$, is simply obtained by integrating equation (A.1), namely:

$$
\bar{v}\left(\Lambda E_{1}\right)=\frac{1}{2}\left[1+\ln \left(\frac{E_{\mathrm{ex}}}{2 \check{E}_{2}}\right)+\frac{1}{2 \check{E}_{2}}-\frac{1}{E_{\mathrm{ex}}}\right]\left(1-\frac{\check{E}_{2}}{\Lambda E_{1}}\right)^{-1}
$$


as shown in figure 11, where it becomes obvious that in the $E_{2}>E_{\text {ex }}$ recoil energy limit a potentially serious overestimation of the displacement damage can occur should we decide to ignore the effect of ionization in the solid.

Considering the case study of $700 \mathrm{keV}$ self-ion irradiation in pure copper the incurred relative error $\Delta \bar{v}\left(\Lambda E_{1}\right) / \bar{v}\left(\Lambda E_{1}\right)$ is about $20 \%$, which is linearly propagated to the displacement cross-section and therefore to the concentration of displaced atoms (or displacement dose) overestimating it by the same amount. In the $E_{2}>E_{\text {ex }}$ recoil energy limit the concentration of displaced atoms (or displacement dose), $C_{\mathrm{d}}=\Phi \sigma_{\mathrm{p}} \bar{v}\left(\Lambda E_{1}\right)$, due to secondary collisions and taking into account the effect of electronic excitation in the solid is then simply defined by:

$$
C_{\mathrm{d}}=\frac{2 \pi \Phi a_{0}^{2} M_{1} Z_{1}^{2} Z_{2}^{2} E_{\mathrm{R}}^{2}}{M_{2} E_{1} \check{E}_{2}}\left[1+\ln \left(\frac{E_{\mathrm{ex}}}{2 \check{E}_{2}}\right)+\frac{1}{2 \check{E}_{2}}-\frac{1}{E_{\mathrm{ex}}}\right] .
$$

\section{References}

Anno J N 1984 Notes on Radiation Effects on Materials (Washington, DC: Hemisphere)

Billington D S and Crawford J H Jr 1961 Radiation Damage in Solids (Princeton, NJ: Princeton University Press)

Chadderton L T 1965 Radiation Damage in Crystals (London: Methuen)

Chaitin G J 1985 An APL2 Gallery of Mathematical-Physics-A Course Outline (Yorktown Heights: IBM Research Division)

Dienes G J and Vineyard G H 1957 Radiation Effects in Solids vol 2 (New York: Interscience)

Golubov S I and Singh B N 2001 Phil. Mag. A 812533

Grenander U 1982 Mathematical Experiments on the Computer (New York: Academic)

Heinisch H L, Greenwood L R, Weber W J and Williford R E 2002 J. Nucl. Mater. 307-311 895

Kinchin G H and Pease R S 1955 Rep. Prog. Phys. 181

Kircher J F 1964 Basic concepts of radiation effects Effects of Radiation on Materials and Components ed J F Kircher and R E Bowman (New York: Reinhold)

Norgett M J, Robinson M T and Torrens I M 1975 Nucl. Eng. Des. 3350

Schilling W and Ullmaier H 1994 Nuclear materials Materials Science and Technology Part II vol 10 B ed R W Cahn, P Haasen and E J Kramer (Basel: VCH)

Sosin A and Bauer W 1969 Atomic displacement mechanism in metals and semiconductors Studies in Radiation Effects in Solids vol 3 ed G J Dienes (New York: Gordon and Breach)

Thompson M W 1969 Defects and Radiation Damage in Metals (Cambridge: Cambridge University Press) 